\section{Mediation and Trust}

\author{
KOME - An International Journal of Pure \\ Communication Inquiry \\ Volume 2 Issue 1 p.1-2 \\ (C) The Author(s) 2014 \\ Reprints and Permission: \\ kome@komejournal.com \\ Published by the Hungarian Communication \\ Studies Assoiciation
}

Zsolt Nagy Károly
Institute of Ethnology, Research Centre for the Humanities of Hungarian Academy of Sciences

The journal KOME, just starting its second volume, is again offering its readers a wide range of texts embracing the field of pure communication inquiry. In the first text of our current issue, Viktor Németh is examining the topic of mediation in the context of the participatory theory of communication. Through analyzing practical examples of mediation, the author presents how this technique can function as the scene of problem solving right through realizing the mutual availability of the preparedness of the participating agents - let them be either individuals or constituents of a coalition - as part of the process. The key element in Németh's interpretation is that the participating agents have the opportunity to reveal their own world for the other party via the contribution of the mediator, and, as the author writes: "As a result, the agents - the existing in two or more own worlds - mutually understanding each other's own reading create a common own world. The own world jointly created this way contains the preparedness, knowledge and skills of all present and having knowledge regarding the topic. In addition, it contains the desired needs and interests as well, which should be met to terminate the problem for the agent. All of the parties concerned have access to the joint preparedness."

In the situations outlined by Németh, trust plays an important role. Its loss is part of the problem, while its recovery, or at least the bridging over its absence via the activity of the mediator is part of the solution to the problem. Losing the trust - or, more precisely, the forfeiture of the trust not yet even gained - is in the background of the processes presented in our second text as well. The baseline of Jim Schnell's study is the situation the U.S. Army has created for itself by ignoring the characteristics of the foreign culture during its operations in Iraq. As the author writes: "Making enemies when we should be making friends created significant obstacles to U.S.-Iraq relations." He thinks this was mostly apparent in that "[e]ach Iraqi owed it to himself and his family to decide whether it made more sense to cooperate with us or to cooperate with somebody else, the insurgents. Unfortunately, because of our incompetence, more and more Iraqis have made the decision that their interests don't lie with us." For solving the problem, as we can read in the text, the army "has deployed" consultants, whose task was to train the American soldiers entering cultural conflicts on the global scene to be some kind of mediators along the straightforward goals and simple methods of a very deliberate strategy. This new knowledge, that is, preparedness, which the author calls cross-cultural competence "provide[s] the ability to operate effectively in any culturally complex environment [...] Cross-cultural competence is recognized as a force multiplier that can significantly enhance operations. It can develop over time through 
experience, but can also be accelerated by principled learning methods. Cross-cultural competence enables negotiation and persuasion; mediation and conflict resolution; leadership and influence; cultural evaluation, synthesis, and predictive analysis during staff planning; and other abilities that pertain to a specific geographic area." Hereafter the faint question arises in the mind of the naive reader trusting the power and potentials of communication: why an army armed by such knowledge still needs rifles, tanks and rockets?

Another type of conflicts and their peculiar solutions are in the focus of Barbara Sólyom's research study, which is rich in empirical data. Sólyom is examining the coexistence of the Hungarian majority and the German ethnic minority in a Hungarian village, particularly the self-defensive strategies of the German minority. She studies the question within the theoretical framework of social psychology, with the concept of social identity in the center. Our social identity is defined from two directions: for one, the way we identify ourselves as the member of a group, and also the way others do this with us. But "we are worried that others may judge us based on stereotypes applied to the group we belong to or that our behavior reinforces these stereotypes. This basically stems from meta-stereotypes: what stereotypes are used regarding the group according to its own individual member. These meta-stereotypes may be the source of social identity threat. They are particularly visible in case of interactions between minorities, notably ethnic groups." Sólyom identifies three strategies the minority members use to defend themselves in the examined situation: firstly, avoiding contact; secondly, dismissing or ignoring the image formed about them by the majority group; while the most complex and riskiest possibility is "coordinating behavior during social interaction. People belonging to the majority or an ethnic minority may also respond to threats against their identity by modifying their behavior in order to ensure a good impression in the eyes of their partners. This means they change their behavior in order to avoid confirming an already existing stereotype and they harmonize it with stereotype expectations applied to the group they belong to." There are several factors determining which option a member of the minority group chooses, but according to Sólyom it is certain that "[t]hose who are motivated to ensure a better image and believe in success also believe that they have the capacities necessary to choose the strategy of controlling behavior." In these conflicts the control of behavior, that is, the intentional cultural "mistranslation," as well as the common reconstruction of reality built on such mistranslations, i.e. stereotypes, evokes the scenes of the majority and the minority groups' coexistence.

The last study of this present issue - contrary to the above - is a theoretical text, in which Márton Demeter drafts a logical system "in which reversible and irreversible processes could be uniformly handled. This attempt originates in the observation that the question of reversibility not essentially emerges in logic, nor yet in temporal logic in spite of the fact that, in principle, it should be an eminent question as regards any action (where time is the vehiculum and presupposition of change). The situation is quite similar with communication theories, where the expression 'communication' usually refers to an individual process or action in spite of the fact that any process presupposes a given, timeless type for this process. This timeless or time-indifferent types are the prototypes of pure communication." Demeter's lucid reasoning is made especially exciting by the diagrammatic representations of the analyzed logical concepts, and not only because this way he promotes a better understanding of the problems - by providing a kind of an illustration - but also because he manages to incorporate the diagrams into the flow of his argument, which in turn leads to a much more complex understanding. 